\title{
One-dimensional superfluid Bose-Fermi mixture: Mixing, demixing, and bright solitons
}

\author{
Sadhan K. Adhikari ${ }^{1, *}$ and Luca Salasnich ${ }^{2, \dagger}$ \\ ${ }^{1}$ Instituto de Física Teórica, UNESP, São Paulo State University, 01.405-900 Sao Paulo, Sao Paulo, Brazil \\ ${ }^{2}$ CNISM and CNR-INFM, Unità di Padova, Dipartimento di Fisica "G. Galilei," Università di Padova, Via Marzolo 8, 35131 Padova, \\ Italy
}

(Received 30 May 2007; published 28 August 2007)

\begin{abstract}
We study an ultracold and dilute superfluid Bose-Fermi mixture confined in a strictly one-dimensional (1D) atomic waveguide by using a set of coupled nonlinear mean-field equations obtained from the Lieb-Liniger energy density for bosons and the Gaudin-Yang energy density for fermions. We consider a finite Bose-Fermi interatomic strength $g_{b f}$ and both periodic and open boundary conditions. We find that with periodic boundary conditions-i.e., in a quasi-1D ring-a uniform Bose-Fermi mixture is stable only with a large fermionic density. We predict that at small fermionic densities the ground state of the system displays demixing if $g_{b f}$ $>0$ and may become a localized Bose-Fermi bright soliton for $g_{b f}<0$. Finally, we show, using variational and numerical solutions of the mean-field equations, that with open boundary conditions-i.e., in a quasi-1D cylinder-the Bose-Fermi bright soliton is the unique ground state of the system with a finite number of particles, which could exhibit a partial mixing-demixing transition. In this case the bright solitons are demonstrated to be dynamically stable. The experimental realization of these Bose-Fermi bright solitons seems possible with present setups.
\end{abstract}

DOI: 10.1103/PhysRevA.76.023612

PACS number(s): 03.75.Ss, 03.75.Hh, 64.75.+g

\section{INTRODUCTION}

The effects of quantum statistics are strongly enhanced in strictly one-dimensional (1D) systems [1]. These effects can be investigated with ultracold and dilute gases of alkalimetal atoms, which are now actively studied in the regime of deep Bose and Fermi degeneracies [2]. Recently two experimental groups $[3,4]$ have reported the observation of the crossover between a 1D quasi-Bose-Einstein condensate (quasi-BEC) in the weak-coupling mean-field GrossPitaevskii (GP) domain and a Tonks-Girardeau (TG) gas $[5,6]$ with ultracold ${ }^{87} \mathrm{Rb}$ atoms in highly elongated traps. A rigorous theoretical analysis of the ground-state properties of a uniform 1D Bose gas was performed by Lieb and Liniger (LL) 44 years ago [6]. An extension of the LL theory for finite and inhomogeneous 1D Bose gases has been proposed on the basis the local density approximation (LDA) [7]. The LDA is improved by including a gradient term that represents additional kinetic energy associated with the inhomogeneity of the gas [8-13].

In the last few years several experimental groups have observed the crossover from the Bardeen-Cooper-Schrieffer (BCS) state of Cooper Fermi pairs to the BEC of molecular dimers with ultracold two-hyperfine-component Fermi vapors of ${ }^{40} \mathrm{~K}$ atoms [14-16] and ${ }^{16} \mathrm{Li}[17,18]$ atoms. It is well known that purely attractive potentials have bound states in $1 \mathrm{D}$ and $2 \mathrm{D}$ for any strength, contrary to the $3 \mathrm{D}$ case [19]. A rigorous theoretical analysis of the ground-state properties of a uniform 1D Fermi gas was performed by Gaudin and Yang (GY) 40 years ago [20]. For repulsive interaction the GY model gives a Tomonaga-Luttinger liquid [21], while for attractive interaction it describes a Luther-Emery (superfluid)

\footnotetext{
*adhikari@ift.unesp.br; URL: www.ift.unesp.br/users/adhikari

†salasnich@pd.infn.it; URL: www.padova.infm.it/salasnich
}

liquid [22]. The ground state of a weakly attractive 1D Fermi gas is a BCS-like state with Cooper pairs, whose size is much larger than the average interparticle spacing. The strong-coupling regime with tightly bound dimers is reached by increasing the magnitude of the attractive interatomic strength. In this regime the fermion pairs behave like a hardcore Bose gas (TG gas) or, equivalently, like 1D noninteracting fermions $[23,24]$.

Degenerate Bose-Fermi mixtures of alkali-metal atoms have been experimentally observed in ${ }^{6,7} \mathrm{Li}[25,26],{ }^{6} \mathrm{Li}^{23} \mathrm{Na}$ [27], and ${ }^{40} \mathrm{~K}-{ }^{87} \mathrm{Rb}$ [28]. In these mixtures, the theoretical investigation of phase separation [29-35] and solitonlike structures has drawn significant attention. Bright solitons have been observed in BECs of $\mathrm{Li}$ [36] and $\mathrm{Rb}$ [37] atoms and studied subsequently [38]. It has been demonstrated using microscopic [39] and mean-field hydrodynamic [40] models that the formation of stable fermionic bright and dark solitons is possible in a degenerate Bose-Fermi mixture as well as in a Fermi-Fermi mixture [41] with the fermions in the normal state in the presence of a sufficiently attractive interspecies interaction which can overcome the Pauli repulsion among fermions. The formation of a soliton in these cases is related to the fact that the system can lower its energy by forming high-density regions (solitons) when the interspecies attraction is large enough to overcome the Pauli repulsion in the degenerate Fermi gas (and any possible repulsion in the BEC) [42]. There have also been studies of the mixing-demixing transition in degenerate Bose-Fermi [43] and Fermi-Fermi [44] mixtures with fermions in the normal state.

After observing the degenerate Fermi gas and the realization of BCS condensed superfluid phase of the fermionic system [45], the BCS-Bose crossover [46] in it seems to be under control [14-18] by manipulating the Fermi-Fermi interaction near a Feshbach resonance [47] by varying a uniform external magnetic field. Naturally, the question of the 
possibility of bright solitons in a superfluid fermionic condensate assisted by a BEC (with attractive interspecies BoseFermi interaction) needs to be revisited. Although the fermionic BCS or molecular dimer phase is possible with an attractive Fermi-Fermi attraction, once formed such a phase is inherently repulsive and self-defocussing and will not lead to a bright soliton spontaneously.

In this paper we investigate a 1D superfluid Bose-Fermi mixture composed of bosonic atoms, well described by the LL theory, and superfluid fermionic atoms, well described by the GY theory with the special intention of studying the localized structures or bright solitons in this superfluid mixture. We derive a set of coupled nonlinear mean-field equations for the Bose-Fermi mixture which we use in the present study. The solution of this mean-field equation is considered two types of boundary conditions: periodic and open. It has been recently shown [48-50] that an attractive BEC with periodic boundary conditions, which can be experimentally produced with a quasi-1D ring [51], displays a quantum phase transition from a uniform state to a symmetry-breaking state characterized by a localized bright-soliton condensate [50]. Here we show that a similar phenomenon appears in the 1D Bose-Fermi mixture with an attractive Bose-Fermi scattering length. Instead, with a repulsive Bose-Fermi scattering length or with the increase of the number of Fermi atoms leading to large repulsion we find a phase separation between bosons and fermions (a mixing-demixing transition), in analogy with previous theoretical [29-35] and experimental studies [25] with $3 \mathrm{D}$ trapped ${ }^{6} \mathrm{Li}-{ }^{7} \mathrm{Li}$ and ${ }^{39} \mathrm{~K}-{ }^{40} \mathrm{~K}$ mixtures. Finally, we predict that with open boundary conditions-i.e., in a infinite quasi-1D cylinder - the ground state of the mixture with sufficiently attractive Bose-Fermi interaction is always a localized bright soliton.

The paper is organized as follows. In Sec. II we describe the 1D model we use in our investigation of the superfluid Bose-Fermi mixture. The Lagrangian density for bosons is appropriate for a TG to BEC crossover through the use of a quasianalytic LL function. The fermions are treated using the GY model through a quasianalytic GY function. The BoseFermi interaction is taken to be a standard contact interaction. The Euler-Lagrange equations for this system are a set of coupled nonlinear mean-field equations which we use in the present study. In Sec. III we consider the system with periodic boundary conditions and obtain the thresholds for demixing for the formation of localized bright solitons (for a sufficiently strong Bose-Fermi attraction) and for the existence of mixing-i.e., states with constant density in space (for weak Bose-Fermi attraction and for Bose-Fermi repulsion). We also present a modulational instability analysis of a uniform solution of the mixture and obtain the condition for the appearance of bright solitons by modulational instability. In Sec. IV we consider the mixture with open boundary conditions and derive a variational approximation for the solution of the mean-field equations of the model using a Gaussian-type ansatz. We investigate numerically the bright Bose-Fermi solitons, demonstrate their stability under perturbation, and compare the numerical results with those of the variational approach. Finally, in Sec. V we present a summary and discussion of our study.

\section{MODEL}

We consider a mixture of $N_{b}$ atomic bosons of mass $m_{b}$ and $N_{f}$ superfluid atomic fermions of mass $m_{f}$ at zero temperature trapped by a tight cylindrically symmetric harmonic potential of frequency $\omega_{\perp}$ in the transverse (radial cylindric) direction. We assume factorization of the transverse degrees of freedom. This is justified in 1D confinement where, regardless of the longitudinal behavior or statistics, the transverse spatial profile is that of the single-particle ground state $[34,52]$. The transverse width of the atom distribution is given by the characteristic harmonic length of the singleparticle ground state: $a_{\perp j}=\sqrt{\hbar /\left(m_{j} \omega_{\perp}\right)}$, with $j=b, f$. The atoms have an effective 1D behavior at zero temperature if their chemical potentials are much smaller than the transverse energy $\hbar \omega_{\perp}$ [34,52]. The boson-boson interaction is described by a contact pseudopotential with repulsive (positive) scattering length $a_{b}$. The fermion-fermion interaction is modeled by a contact pseudopotential with attractive (negative) scattering length $a_{f}$. The boson-fermion interaction is instead characterized by a contact pseudopotential with scattering length $a_{b f}$, which can be repulsive or attractive. To avoid the confinement-induced resonance [53] we take $a_{b},\left|a_{f}\right|,\left|a_{b f}\right| \ll a_{\perp b}, a_{\perp f}$.

We use a mean-field effective Lagrangian to study the static and collective properties of the 1D Bose-Fermi mixture. The Lagrangian density $\mathcal{L}$ of the mixture reads

$$
\mathcal{L}=\mathcal{L}_{b}+\mathcal{L}_{f}+\mathcal{L}_{b f}
$$

The term $\mathcal{L}_{b}$ is the bosonic Lagrangian, defined as

$$
\mathcal{L}_{b}=i \hbar \psi_{b}^{*} \frac{\partial}{\partial t} \psi_{b}+\frac{\hbar^{2}}{2 m_{b}} \psi_{b}^{*} \frac{\partial^{2}}{\partial z^{2}} \psi_{b}-\frac{\hbar^{2}}{2 m_{b}}\left|\psi_{b}\right|^{6} G\left(\frac{m_{b} g_{b}}{\hbar^{2}\left|\psi_{b}\right|^{2}}\right),
$$

where $\psi_{b}(z, t)$ is the hydrodynamic field of the Bose gas along the longitudinal axis, such that $n_{b}(z, t)=\left|\psi_{b}(z, t)\right|^{2}$ is the 1D local probability density of bosonic atoms and $g_{b}$ $=2 \hbar \omega_{\perp} a_{b}$ is the $1 \mathrm{D}$ boson-boson interaction strength $\left(g_{b}\right.$ $>0) . G(x)$ is the Lieb-Liniger function [54], defined as the solution of a Fredholm equation for $x>0$ and such that [6]

$$
G(x)=\left\{\begin{array}{cc}
x-\frac{4}{3 \pi} x^{3 / 2}+\cdots & \text { for } 0<x \ll 1, \\
\frac{\pi^{2}}{3}\left(1-\frac{2}{x}+\cdots\right) & \text { for } x \gg 1 .
\end{array}\right.
$$

In the extreme weak-coupling limit $x \rightarrow+0$, and $G(x) \rightarrow x$ and, consequently, the bosonic Lagrangian above reduces to the standard mean-field GP Lagrangian. In the static case the Lagrangian density $\mathcal{L}_{b}$ reduces exactly to the energy functional recently introduced by Lieb, Seiringer, and Yngvason [9]. In addition, $\mathcal{L}_{b}$ has been successfully used to determine the collective oscillation of the 1D Bose gas with longitudinal harmonic confinement [13]. In the strong-coupling limit $x \rightarrow+\infty$ and $G(x) \rightarrow \pi^{2} / 3$ while the Lagrangian above reduces to the strongly repulsive bosonic Lagrangian in the TG limit [5]. As $x$ changes from +0 to $+\infty$ the above Lagrangian shows a continuous transition from the GP BEC to TG phase. 
The fermionic Lagrangian density $\mathcal{L}_{f}$ is given instead by

$$
\mathcal{L}_{f}=i \hbar \psi_{f}^{*} \frac{\partial}{\partial t} \psi_{f}+\frac{\hbar^{2}}{2 m_{f}} \psi_{f}^{*} \frac{\partial^{2}}{\partial z^{2}} \psi_{f}-\frac{\hbar^{2}}{2 m_{f}}\left|\psi_{f}\right|^{6} F\left(\frac{m_{f} g_{f}}{\hbar^{2}\left|\psi_{f}\right|^{2}}\right),
$$

where $\psi_{f}(z, t)$ is the field of the 1D superfluid Fermi gas, such that $n_{f}(z, t)=\left|\psi_{f}(z, t)\right|^{2}$ is the 1D fermionic local density along the longitudinal axis and $g_{f}=2 \hbar \omega_{\perp} a_{f}$ is the 1D fermion-fermion interaction strength $\left(g_{f}<0\right) . F(x)$ is the Gaudin-Yang function [55], defined as the solution of a Fredholm equation for $x<0$ and such that $[20,23]$

$$
F(x)=\left\{\begin{array}{cc}
\frac{\pi^{2}}{48}\left(1-\frac{1}{x}+\frac{3}{4 x^{2}}+\cdots\right) & \text { for } x \ll-1, \\
\frac{\pi^{2}}{12}\left(1+\frac{6}{\pi^{2}} x+\cdots\right) & \text { for }-1 \ll x<0 .
\end{array}\right.
$$

In the static and uniform case the Lagrangian density $\mathcal{L}_{f}$ reduces exactly to Gaudin-Yang energy functional [20], which has been recently used by Fuchs et al. [23].

The physical content of the fermionic Lagrangian (4) is easy to understand. For $x \rightarrow-0$ we are in the domain of weak Fermi-Fermi attraction $\left(a_{f}, g_{f} \rightarrow 0\right.$, corresponding to the BCS limit), while $F(x) \rightarrow \pi^{2} / 12$ from Eq. (5). Consequently, the fermionic interaction term in Eq. (4) involving the $F(x)$ function reduces to $\left(\hbar^{2} / 2 m_{f}\right)\left(\pi^{2} / 12\right)\left|\psi_{f}\right|^{6}$ (independent of FermiFermi interaction strength $g_{f}$ or Fermi-Fermi scattering length $a_{f}$ ), which is the Pauli repulsive term of noninteracting fermions considered in Refs. $[52,56]$ arising due to the Pauli exclusion principle and not due to the fundamental Fermi-Fermi interaction implicit in the scattering length $a_{f}$ via $g_{f}$. This is quite expected as by taking the $x \rightarrow-0$ limit we switch off the Fermi-Fermi attraction and pass from the BCS domain to a degenerate noninteracting Fermi gas (in nonsuperfluid phase) studied in Ref. [52] corresponding to $x \rightarrow+0$. [Although the probability densities in the $x \rightarrow+0$ (nonsuperfluid degenerate Fermi gas) and $x \rightarrow-0$ (superfluid BCS condensate) limits are identical, they correspond to different physical states. The superfluid phase gas a "gap" associated with it describable by the BCS equation.] As $x$ passes from -0 to $-\infty$, the Fermi-Fermi attraction increases and we move from the superfluid BCS regime of weakly bound Cooper pairs to the unitarity regime of strongly-bound molecular fermions. (The unitarity limit corresponds to infinitely large Fermi-Fermi attraction: $a_{f} \rightarrow-\infty$.) As the FermiFermi scattering length $\left|a_{f}\right|$ increases from the BCS to the unitarity limit, the interaction energy in Eq. (4) becomes a term dependent on $a_{f}$. However, in the unitarity limit $a_{f} \rightarrow$ $-\infty$ and all the fermions will be paired to form strongly bound noninteracting molecules and the Fermi-Fermi interaction term in Eq. (4) behaves like that of a Tonks-Girardeau gas of bosons-bosonic molecules - and again becomes independent of $a_{f}$ [23]. The system then becomes a Bose gas of molecules and its interaction energy is no longer a function of $a_{f}$.
Finally, the Lagrangian density $\mathcal{L}_{b f}$ of the Bose-Fermi interaction taken to be of the following standard zero-range form $[43,52]$

$$
\mathcal{L}_{b f}=-g_{b f}\left|\psi_{b}\right|^{2}\left|\psi_{f}\right|^{2},
$$

where $g_{b f}=2 \hbar \omega_{\perp} a_{b f}$ is the $1 \mathrm{D}$ boson-fermion interaction strength.

The Euler-Lagrange equations of the Lagrangian $\mathcal{L}$ are the two following coupled partial differential equations:

$$
\begin{aligned}
i \hbar \partial_{t} \psi_{b}= & {\left[-\frac{\hbar^{2}}{2 m_{b}} \partial_{z}^{2}+3 \frac{\hbar^{2}}{2 m_{b}} n_{b}^{2} G\left(\frac{m_{b} g_{b}}{\hbar^{2} n_{b}}\right)-\frac{1}{2} g_{b} n_{b} G^{\prime}\left(\frac{m_{b} g_{b}}{\hbar^{2} n_{b}}\right)\right.} \\
& \left.+g_{b f} n_{f}\right] \psi_{b}, \\
i \hbar \partial_{t} \psi_{f}= & {\left[-\frac{\hbar^{2}}{2 m_{f}} \partial_{z}^{2}+3 \frac{\hbar^{2}}{2 m_{f}} n_{f}^{2} F\left(\frac{m_{f} g_{f}}{\hbar^{2} n_{f}}\right)-\frac{1}{2} g_{f} n_{f} F^{\prime}\left(\frac{m_{f} g_{f}}{\hbar^{2} n_{f}}\right)\right.} \\
& \left.+g_{b f} n_{b}\right] \psi_{f},
\end{aligned}
$$

where $n_{j}=\left|\psi_{j}\right|^{2}, j=b, f$, are probability densities for bosons and fermions, respectively, with the normalization $\int_{-\infty}^{\infty} n_{j} d z$. $=N_{j}$.

It is convenient to work in terms of dimensionless variables defined in terms of a frequency $\omega$ and length $l$ $\equiv \sqrt{\hbar /\left(m_{b} \omega\right)}$ by $\psi_{j}=\hat{\psi}_{j} / \sqrt{l}, t=2 \hat{t} / \omega, z=\hat{z} l, g_{j}=\hat{g}_{j} \hbar^{2} /\left(2 m_{b} l\right)$, and $g_{b f}=\hat{g}_{b f} \hbar^{2} /\left(2 m_{b} l\right)$. In terms of these new variables Eqs. (7) and (8) can be written as

$$
\begin{aligned}
i \partial_{t} \psi_{b} & =\left[-\partial_{z}^{2}+3 n_{b}^{2} G\left(\frac{g_{b}}{2 n_{b}}\right)-\frac{1}{2} g_{b} n_{b} G^{\prime}\left(\frac{g_{b}}{2 n_{b}}\right)+g_{b f} n_{f}\right] \psi_{b}, \\
i \partial_{t} \psi_{f} & =\left[-\lambda \partial_{z}^{2}+3 \lambda n_{f}^{2} F\left(\frac{g_{f}}{2 n_{f}}\right)-\frac{1}{2} g_{f} n_{f} F^{\prime}\left(\frac{g_{f}}{2 n_{f}}\right)+g_{b f} n_{b}\right] \psi_{f},
\end{aligned}
$$

where we have dropped the carets over the variables and where $\lambda=m_{b} / m_{f}, n_{j}=\left|\psi_{j}\right|^{2}, j=b, f$, are probability densities for bosons and fermions, respectively, with the normalization $\int_{-\infty}^{\infty} n_{j} d z=N_{j}$. However, in the present study we set $\lambda=1$ in the following. All results reported in this paper are for this case. This should approximate well the Bose-Fermi mixtures ${ }^{7} \mathrm{Li}-{ }^{6} \mathrm{Li}$ and ${ }^{39} \mathrm{~K}-{ }^{40} \mathrm{~K}$ of experimental interest.

The bosonic and fermionic nonlinearities in Eqs. (9) and (10), respectively, have complex structures in general. However, in the weak-coupling GP limit $\left(g_{b} \rightarrow 0\right)$ the bosonic nonlinearity in Eq. (9) is cubic and turns quintic in the strong-coupling TG limit $\left(g_{b} \rightarrow+\infty\right)$. The fermionic nonlinearity in Eq. (10) becomes quintic in both weak $\left(g_{F} \rightarrow-0\right)$ and strong $\left(g_{f} \rightarrow-\infty\right)$ coupling but with coefficients $\pi^{2} / 12$ and $\pi^{2} / 48$, respectively.

For stationary states the solutions of Eqs. (9) and (10) have the form $\psi_{j}=\phi_{j} \exp \left(-i \mu_{j} t\right)$ where $\mu_{j}$ are the respective chemical potentials. Consequently, these equations reduce to 


$$
\begin{gathered}
\mu_{b} \phi_{b}=\left[-\partial_{z}^{2}+3 n_{b}^{2} G\left(\frac{g_{b}}{2 n_{b}}\right)-\frac{1}{2} g_{b} n_{b} G^{\prime}\left(\frac{g_{b}}{2 n_{b}}\right)+g_{b f} n_{f}\right] \phi_{b}, \\
\mu_{f} \phi_{f}=\left[-\partial_{z}^{2}+3 n_{f}^{2} F\left(\frac{g_{f}}{2 n_{f}}\right)-\frac{1}{2} g_{f} n_{f} F^{\prime}\left(\frac{g_{f}}{2 n_{f}}\right)+g_{b f} n_{b}\right] \phi_{f} .
\end{gathered}
$$

A repulsive Bose-Bose interaction is produced by a positive $g_{b}$, while an attractive Fermi-Fermi interaction corresponds to a negative $g_{f}$.

\section{MIXTURE WITH PERIODIC BOUNDARY CONDITIONS}

\section{A. General considerations}

Here we consider the 1D Bose-Fermi mixture with periodic boundary conditions. These boundary conditions can be used to model a quasi-1D ring of radius $R$. If the radius is much larger than the transverse width-i.e., $R \gg a_{\perp j}$, $j=b, f$-then effects of curvature can be neglected [50] and one can safely use the Lagrangian density (1) with $z=R \theta$, where $\theta$ the azimuthal angle [50,51]. The energy density of the uniform mixture is immediately derived from the Lagrangian density (1), with Eqs. (2), (4), and (6), dropping out space-time derivatives. It is given by

$$
\mathcal{E}=n_{b}^{3} G\left(\frac{g_{b}}{2 n_{b}}\right)+n_{f}^{3} F\left(\frac{g_{f}}{2 n_{f}}\right)+g_{b f} n_{b} n_{f} .
$$

In this case one can have a uniform mixture or the formation of solitonlike structures and in the following we study the condition for these possibilities to happen. Obviously, for the 1D uniform mixture in the ring of radius $R$, we have $n_{b}$ $=N_{b} /(2 \pi R)$ and $n_{f}=N_{f} /(2 \pi R)$.

The uniformly mixed phase is energetically stable if its energy is a minimum with respect to small variations of the densities $n_{f}$ and $n_{b}$, while the total number of fermions and bosons are held fixed. To get the equilibrium densities one must then minimize the function

$$
\widetilde{\mathcal{E}}\left(n_{b}, n_{f}\right)=\mathcal{E}\left(n_{b}, n_{f}\right)-\mu_{b}\left(n_{b}^{*}, n_{f}^{*}\right) n_{b}-\mu_{f}\left(n_{b}^{*}, n_{f}^{*}\right) n_{f},
$$

where $\mu_{b}$ and $\mu_{f}$ are Lagrange multipliers (imposing that the numbers of bosons and fermions are fixed) which may be identified with the Bose and Fermi chemical potentials and $n_{b}^{*}$ and $n_{f}^{*}$ are the values of $n_{b}$ and $n_{f}$ at the minimum. Setting the derivatives of $\widetilde{\mathcal{E}}$ with respect to $n_{f}$ and $n_{b}$ equal to zero, one finds

$$
\begin{gathered}
\mu_{b}=\frac{\partial \mathcal{E}}{\partial n_{b}}=3 n_{b}^{2} G\left(\frac{g_{b}}{2 n_{b}}\right)-\frac{1}{2} g_{b} n_{b} G^{\prime}\left(\frac{g_{b}}{2 n_{b}}\right)+g_{b f} n_{f}, \\
\mu_{f}=\frac{\partial \mathcal{E}}{\partial n_{f}}=3 n_{f}^{2} F\left(\frac{g_{f}}{2 n_{f}}\right)-\frac{1}{2} g_{f} n_{f} F^{\prime}\left(\frac{g_{f}}{2 n_{f}}\right)+g_{b f} n_{b} .
\end{gathered}
$$

The solution of Eqs. (16) and (15), which are exactly Eqs. (11) and (12) if one drops out the space derivatives, gives a
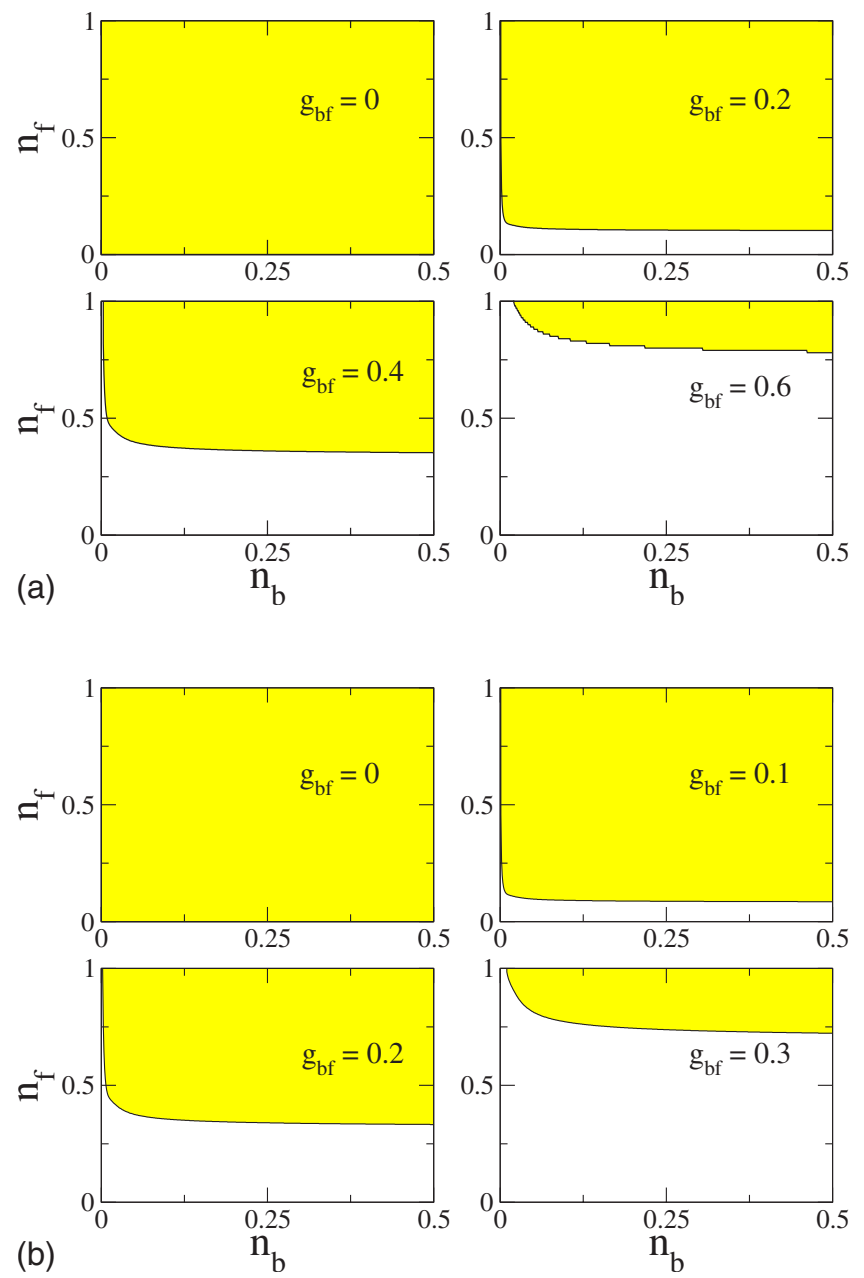

FIG. 1. (Color online) Region of stability of the homogeneous mixture denoted by shaded (gray) area in the plane $\left(n_{b}, n_{f}\right)$. Different panels correspond to different values of the Bose-Fermi interaction strength $g_{b f}$. We set $g_{b}=0.1$ and (a) $g_{f}=-0.1$ (corresponding to a weak Fermi-Fermi attraction in the BCS phase) and (b) $g_{f}=-30$ (corresponding to a strong Fermi-Fermi attraction, with fermions in the unitarity regime).

minimum if the corresponding Hessian of $\widetilde{\mathcal{E}}$ is positive-i.e., if

$$
\frac{\partial^{2} \tilde{\mathcal{E}}}{\partial n_{b}^{2}} \frac{\partial^{2} \mathcal{E}}{\partial n_{f}^{2}}-\left(\frac{\partial^{2} \widetilde{\mathcal{E}}}{\partial n_{b} \partial n_{f}}\right)^{2}>0 .
$$

The solution of this inequality gives the region in the parameters' space where the homogeneous mixed phase is energetically stable.

In Fig. 1 we show the region of stability of the homogeneous mixture by the shaded (gray) area in the plane $\left(n_{b}, n_{f}\right)$ for different values of $g_{b f}$. Note that the sign of $g_{b f}$ is not important because, in Eq. (17), $g_{b f}^{2}$ appears. The figure shows that, by increasing $g_{b f}$, the uniform mixture is stable only at large fermionic densities $n_{f}$. This result, which is in agreement with previous 1D predictions [34] on a 1D mixture of Bose-condensed atoms and normal Fermi atoms, is exactly the opposite of what happens in a 3D mixture of bosons and 

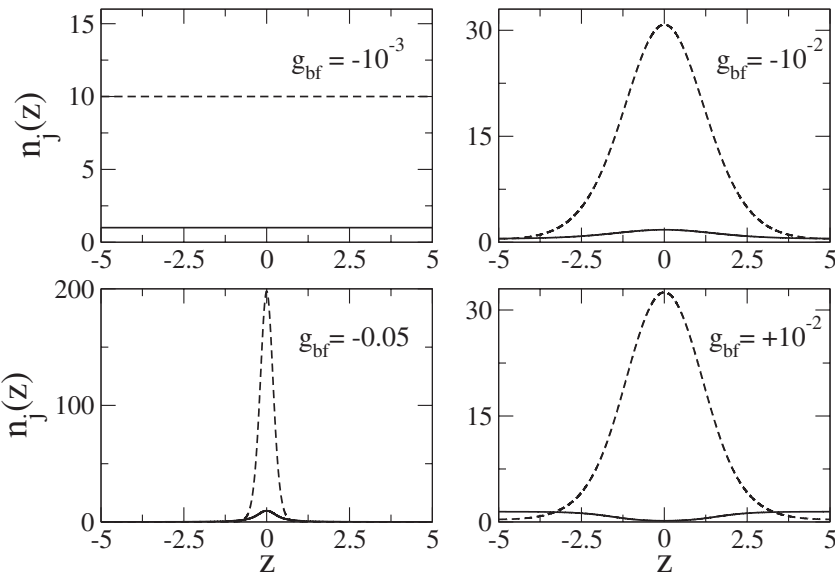

FIG. 2. Probability density profiles $n_{b}(z)$ of bosons (dashed line) and $n_{f}(z)$ of fermions (solid line) of the Bose-Fermi mixture with $N_{b}=100$ bosons and $N_{f}=10$ fermions and periodic boundary conditions (axial length $L \equiv 2 \pi R=10$ ). We choose $g_{b}=0.01$ and $g_{f}=-0.025$ and calculate the profiles for different values of the Bose-Fermi interaction strength $g_{b f}$.

fermions. In fact, a uniform 3D mixture is stable only for small values of the fermionic density [29-33,35]. Figure 1 also shows that when bosons enter in the TG regime (i.e., $\left.g_{b} / n_{b} \gg 1\right)$ the uniform mixture is much less stable: see the behavior of the stability line at very small $n_{b}$, with $g_{b f} \neq 0$.

The panels of Fig. 1 suggest that, with a finite $g_{b f}$, at small fermionic densities the uniform mixture is unstable: the ground state of the system displays demixing if $g_{b f}>0$ and becomes a localized Bose-Fermi bright soliton if $g_{b f}<0$. These effects are clearly shown in Fig. 2, where we plot the density profiles $n_{j}(z)$, with $j=b, f$, for different values of $g_{b f}$ calculated by directly solving Eqs. (11) and (12) numerically with appropriate boundary conditions.

The profiles of probability densities plotted in Fig. 2 demonstrate the uniform-to-localized and the uniform-todemixed transitions. In fact, a large and negative $g_{b f}$ induces a strong localization while a large and positive $g_{b f}$ produces demixing. These density profiles have been obtained by solving Eqs. (9) and (10) with a finite-difference CrankNicholson algorithm and imaginary time [57].

In the regime $g_{b} / n_{b} \ll 1$ (BEC limit) and $\left|g_{f}\right| / n_{f} \ll 1$ (BCS limit), the above analysis yields simple analytic results. In this case $\mathcal{E}=g_{b} n_{b}^{2} / 2+\pi^{2} n_{f}^{3} / 12+g_{b f} n_{b} n_{f}$, consequently, Eq. (17) leads to the condition

$$
\frac{1}{2} \pi^{2} g_{b} n_{f}>g_{b f}^{2}
$$

for the stability of the uniform mixture. The condition $\pi^{2} g_{b} n_{f} / 2>g_{b f}^{2}$ is qualitatively consistent with Fig. 1(a). For example, for $g_{b}=0.1$ and $g_{f}=-0.1$ the condition of stability of uniform mixture becomes $n_{f}>2 g_{b f}^{2}$ and for $\left|g_{b f}\right|=0,0.2$, 0.4 , and 0.6 leads to $n_{f}>0,0.08,0.32$, and 0.72 , respectively.

In the regime $g_{b} / n_{b} \ll 1$ (BEC limit) and $\left|g_{f} / n_{f}\right| \gg 1$ (unitarity or tightly bound molecule formation limit) $\mathcal{E}=g_{b} n_{b}^{2} / 2$ $+\pi^{2} n_{f}^{3} / 48+g_{b f} n_{b} n_{f}$, consequently, Eq. (17) leads to the condition

$$
\frac{1}{8} \pi^{2} g_{b} n_{f}>g_{b f}^{2}
$$

for the stability of the uniform mixture. For example, for $g_{b}=0.1$ and $g_{f}=-30$ the condition of stability of uniform mixture becomes $n_{f}>8 g_{b f}^{2}$ and for $\left|g_{b f}\right|=0,0.1,0.2$, and 0.3 leads to $n_{f}>0,0.08,0.32$, and 0.72 , respectively, in qualitative agreement with Fig. 1(b). The time-independent conditions (18) and (19) are also derived in following subsection using rigorous time-dependent modulational instability analysis of the uniform mixture (constant-amplitude solutions) in the weak and strong Fermi-Fermi coupling limits.

Finally, in the regime $g_{b} / n_{b} \gg 1$ (TG limit) and $\left|g_{f} / n_{f}\right|$ $\ll 1$ (BCS limit) $\mathcal{E}=\pi^{2} n_{b}^{3} / 3+\pi^{2} n_{f}^{3} / 12+g_{b f} n_{b} n_{f}$, consequently, Eq. (17) leads to the condition

$$
\pi^{4} n_{b} n_{f}>g_{b f}^{2}
$$

for the stability of the uniform mixture. The functional dependence of condition (20) on $n_{b}, n_{f}, g_{b}$, and $g_{f}$ is qualitatively different from conditions (18) and (19).

The inequality (17) can be written as

$$
c_{b}^{2} c_{f}^{2}>4 g_{b f}^{2} n_{b} n_{f},
$$

where $c_{f}=\sqrt{2 n_{f}\left(\partial \mu_{f} / \partial n_{f}\right)}$ is the sound velocity of the superfluid Fermi component and $c_{b}=\sqrt{2 n_{b}\left(\partial \mu_{b} / \partial n_{b}\right)}$ is the sound velocity of the Bose gas. The sound velocity $c_{b f}$ of the $1 \mathrm{D}$ Fermi-Bose mixture can be easily obtained following a procedure similar to the one suggested by Alexandrov and Kabanov [58] for a two-component BEC. One finds

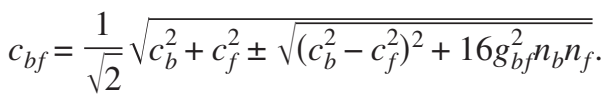

Thus the sound velocity has two branches and the homogeneous mixture becomes dynamically unstable when the lower branch becomes imaginary.

\section{B. Modulational instability}

We show in the following that for attractive Bose-Fermi interaction, the transition from uniform mixture to localized solitonlike structures considered in Sec. III A is due to modulational instability. To study analytically the modulational instability $[59,60]$ of Eqs. (9) and (10) we consider the special case of weak Bose-Bose (small positive $g_{b}$ ) and both weak (small $\left|g_{f} / n_{f}\right| \ll 1$ corresponding to BCS limit) and strong (large $\left|g_{f} / n_{f}\right| \gg 1$ corresponding to molecular unitarity limit) Fermi-Fermi interactions while these equations reduce to

$$
\begin{gathered}
i \partial_{t} \psi_{b}=\left[-\partial_{z}^{2}+g_{b}\left|\psi_{b}\right|^{2}-g_{b f}\left|\psi_{f}\right|^{2}\right] \psi_{b}, \\
i \partial_{t} \psi_{f}=\left[-\partial_{z}^{2}+\kappa \pi^{2}\left|\psi_{f}\right|^{4}-g_{b f}\left|\psi_{b}\right|^{2}\right] \psi_{f},
\end{gathered}
$$

where $\kappa=1 / 4$ for $\left|g_{f} / n_{f}\right| \ll 1$ and $1 / 16$ for $\left|g_{f} / n_{f}\right| \gg 1$. Here we have taken the interspecies interaction to be attractive by inserting an explicit negative sign in $g_{b f}$.

We analyze the modulational instability of a constantamplitude solution corresponding to a uniform mixture in coupled equations (23) and (24) by considering the solutions 


$$
\begin{aligned}
& \varphi_{b 0}=A_{b 0} \exp \left(i \delta_{b}\right) \equiv A_{b 0} e^{i t\left(g_{b f} A_{f 0}^{2}-g_{b} A_{b 0}^{2}\right)}, \\
& \varphi_{f 0}=A_{f 0} \exp \left(i \delta_{f}\right) \equiv A_{f 0} e^{i t\left(g_{b f} A_{b 0}^{2}-\kappa \pi^{2} A_{f 0}^{4}\right)},
\end{aligned}
$$

of Eqs. (23) and (24), respectively, where $A_{j 0}$ is the amplitude and $\delta_{j}$ a phase for component $j=b, f$. The constantamplitude solution develops an amplitude-dependent phase on time evolution. We consider a small perturbation $A_{j} \exp \left(i \delta_{j}\right)$ to these solutions via

$$
\varphi_{j}=\left(A_{j 0}+A_{j}\right) \exp \left(i \delta_{j}\right),
$$

where $A_{j}=A_{j}(z, t)$. Substituting these perturbed solutions in Eqs. (23) and (24) and for small perturbations retaining only the linear terms in $A_{j}$, we get

$$
\begin{gathered}
i \partial_{t} A_{b}+\partial_{z}^{2} A_{b}-g_{b} A_{b 0}^{2}\left(A_{b}+A_{b}^{*}\right)+g_{b f} A_{b 0} A_{f 0}\left(A_{f}+A_{f}^{*}\right)=0, \\
i \partial_{t} A_{f}+\partial_{z}^{2} A_{f}-2 \kappa \pi^{2} A_{f 0}^{4}\left(A_{f}+A_{f}^{*}\right)+g_{b f} A_{b 0} A_{f 0}\left(A_{f}+A_{f}^{*}\right)=0 .
\end{gathered}
$$

We consider the complex plane-wave perturbation

$$
A_{j}(z, t)=\mathcal{A}_{j 1} \cos (K t-\Omega z)+i \mathcal{A}_{j 2} \sin (K t-\Omega z),
$$

with $j=b, f$, where $\mathcal{A}_{j 1}$ and $\mathcal{A}_{j 2}$ are the amplitudes for the real and imaginary parts, respectively, and $K$ and $\Omega$ are frequency and wave numbers.

Substituting Eq. (30) into Eqs. (28) and (29) and separating the real and imaginary parts we get

$$
\begin{gathered}
-\mathcal{A}_{b 1} K=\mathcal{A}_{b 2} \Omega^{2}, \\
-\mathcal{A}_{b 2} K=\mathcal{A}_{b 1} \Omega^{2}-2 g_{b f} A_{b 0} A_{f 0} \mathcal{A}_{f 1}+2 g_{b} A_{b 0}^{2} \mathcal{A}_{b 1},
\end{gathered}
$$

for $j=b$, and

$$
\begin{gathered}
-A_{f 1} K=A_{f 2} \Omega^{2}, \\
-\mathcal{A}_{f 2} K=\mathcal{A}_{f 1} \Omega^{2}-2 g_{b f} A_{b 0} A_{f 0} \mathcal{A}_{b 1}+4 \kappa \pi^{2} A_{f 0}^{4} A_{f 1},
\end{gathered}
$$

for $j=f$. Eliminating $\mathcal{A}_{b 2}$ between Eqs. (31) and (32) we get

$$
A_{b 1}\left[K^{2}-\Omega^{2}\left(\Omega^{2}+2 g_{b} A_{b 0}^{2}\right)\right]=-2 \mathcal{A}_{f 1} g_{b f} A_{b 0} A_{f 0} \Omega^{2}
$$

and eliminating $\mathcal{A}_{f 2}$ between (33) and (34), we have

$$
\mathcal{A}_{f 1}\left[K^{2}-\Omega^{2}\left(\Omega^{2}+4 \kappa \pi^{2} A_{f 0}^{4}\right)\right]=-2 \mathcal{A}_{b 1} g_{b f} A_{b 0} A_{f 0} \Omega^{2} .
$$

Finally, eliminating $\mathcal{A}_{b 1}$ and $\mathcal{A}_{f 1}$ from Eqs. (35) and (36), we obtain the dispersion relation

$$
\begin{aligned}
K= & \pm \Omega\left[\left(\Omega^{2}+g_{b} A_{b 0}^{2}+2 \kappa \pi^{2} A_{f 0}^{4}\right) \pm\left\{\left(g_{b} A_{b 0}^{2}-2 \kappa \pi^{2} A_{f 0}^{4}\right)^{2}\right.\right. \\
& \left.\left.+4 g_{b f}^{2} A_{b 0}^{2} A_{f 0}^{2}\right\}^{1 / 2}\right]^{1 / 2}
\end{aligned}
$$

For stability of the plane-wave perturbation, $K$ has to be real. For any $\Omega$ this happens for

$$
\left(g_{b} A_{b 0}^{2}+2 \kappa \pi^{2} A_{f 0}^{4}\right)^{2}>\left(g_{b} A_{b 0}^{2}-2 \kappa \pi^{2} A_{f 0}^{4}\right)^{2}+4 g_{b f}^{2} A_{b 0}^{2} A_{f 0}^{2}
$$

or for

$$
2 g_{b} \kappa \pi^{2} A_{f 0}^{2}>g_{b f}^{2}
$$

However, for $2 g_{b} \kappa \pi^{2} A_{f 0}^{2}<g_{b f}^{2}, K$ can become imaginary and the plane-wave perturbation can grow exponentially with time. This is the domain of modulational instability of a constant-amplitude solution (uniform mixture of Sec. III A), signaling the possibility of coupled Bose-Fermi bright soliton to appear. Noting that $A_{f 0}^{2}=n_{f}$ for the uniform mixture, this result is consistent with the stability analysis of Sec. III A. In the weak-coupling BCS limit $\kappa=1 / 4$ and Eq. (39) reduces to Eq. (18) obtained from energetic considerations. In the strong-coupling unitarity limit $\kappa=1 / 16$ and Eq. (39) reduces to Eq. (19) for the stability of the uniform mixture. Finally, we comment that Eq. (20) can also be derived in a straightforward fashion from the modulational instability analysis of an appropriate set of dynamical equations, with $g_{b}\left|\psi_{b}\right|^{2}$ replaced by $\pi^{2}\left|\psi_{b}\right|^{4}$ in Eq. (23) and $\kappa=1 / 4$ in Eq. (24).

\section{MIXTURE WITH OPEN BOUNDARY CONDITIONS}

In this section we consider the 1D mixture with open boundary conditions. These boundary conditions can be used to model a quasi-1D cylinder. In practice, if the axial length $L$ of the atomic waveguide is much larger than the transverse width-i.e., $L \gg a_{\perp j}, j=b, f$-then one has a quasi-1D cylinder. The quasi-1D cylinder becomes infinite for $L \rightarrow \infty$. It this case we can use the Lagrangian density (1) with $z \in(-\infty$, $+\infty)$. With a finite number of particles (bosons and fermions) the uniform mixture in a infinite cylinder cannot exist, but localized solutions are instead possible with an attractive Bose-Fermi strength $\left(g_{b f}<0\right)[52]$.

\section{A. Variational results}

Here we develop a variational localized solution to Eqs. (11) and (12), noting that these equations can be derived from the Lagrangian [61]

$$
\begin{aligned}
L= & \int_{-\infty}^{\infty}\left[\mu_{b} \phi_{b}^{2}+\mu_{f} \phi_{f}^{2}-\left(\phi_{b}^{\prime}\right)^{2}-\left(\phi_{f}^{\prime}\right)^{2}-\phi_{b}^{6} G\left(g_{b} / 2 \phi_{b}^{2}\right)\right. \\
& \left.-\phi_{f}^{6} F\left(g_{f} / 2 \phi_{f}^{2}\right)-g_{b f} \phi_{b}^{2} \phi_{f}^{2}\right] d z-\mu_{b} N_{b}-\mu_{f} N_{f},
\end{aligned}
$$

by demanding $\delta L / \delta \phi_{b}=\delta L / \delta \phi_{f}=\delta L / \delta \mu_{b}=\delta L / \delta \mu_{f}=0$.

To develop the variational approximation we use the Gaussian ansatz [62]

$$
\begin{aligned}
& \phi_{b}(z)=\pi^{-1 / 4} \sqrt{\frac{N_{b} A_{b}}{w_{b}}} \exp \left(-\frac{z^{2}}{2 w_{b}^{2}}\right), \\
& \phi_{f}(z)=\pi^{-1 / 4} \sqrt{\frac{N_{f} A_{f}}{w_{f}}} \exp \left(-\frac{z^{2}}{2 w_{f}^{2}}\right),
\end{aligned}
$$

where the variational parameters are $A_{j}$, the solitons' norm and $w_{j}$ the widths, in addition to $\mu_{j}$. Note that this Gaussian ansatz can be extended to include time dependence [63], as done recently to investigate the collective oscillation of a quasi-1D mixture made of condensed bosons and normal fermions [52]. 
The substitution of this variational ansatz in Lagrangian (40) yields

$$
\begin{aligned}
L= & \mu_{b} N_{b} A_{b}+\mu_{f} N_{f} A_{f}-\frac{N_{b} A_{b}}{2 w_{b}^{2}}-\frac{N_{f} A_{f}}{2 w_{f}^{2}}-\frac{A_{b}^{3} N_{b}^{3}}{\pi \sqrt{3} w_{b}^{2}} G\left(\frac{c g_{b} w_{b}}{2 N_{b} A_{b}}\right) \\
& -\frac{A_{f}^{3} N_{f}^{3}}{\sqrt{3} \pi w_{f}^{2}} F\left(\frac{d g_{f} w_{f}}{2 N_{f} A_{f}}\right)-\frac{g_{b f} N_{b} N_{f} A_{b} A_{f}}{\sqrt{\pi\left(w_{f}^{2}+w_{b}^{2}\right)}}-\mu_{b} N_{b}-\mu_{f} N_{f},
\end{aligned}
$$

with $c=\sqrt{3 \pi / 2}$. The integrals over the $G(x)$ and $F(x)$ functions cannot be evaluated exactly for all $x$. However, the term involving the LL $G(x)$ function can be evaluated analytically in the GP $(x \rightarrow 0)$ and TG $(x \gg 1)$ limits. The analytic term involving the $G(x)$ function in Eq. (43) is exact in both the limits and provides a good description of the integral at other values of argument provided we take for the constant $c$ $=\sqrt{3 \pi / 2}$. The fermionic integral over the GY function $F(x)$ is evaluated similarly. There is no obvious reason for choosing the constant $d$ in this integral. After a little experimentation it is taken to be $d=c=\sqrt{3 \pi / 2}$ which provides a faithful analytical representation of the numerical result.

The first variational equations emerging from Eq. (43) $\partial L / \partial \mu_{b}=\partial L / \partial \mu_{f}=0$ yield $A_{b}=A_{f}=1$. Therefore the conditions $A_{b}=A_{f}=1$ will be substituted in the subsequent variational equations. The variational equations $\partial L / \partial w_{j}=0$ lead to

$$
\begin{aligned}
1 & +\frac{2 N_{b}^{2}}{\pi \sqrt{3}} G\left(\frac{c g_{b} w_{b}}{2 N_{b}}\right)-\frac{N_{b} g_{b} w_{b}}{2 \sqrt{2 \pi}} G^{\prime}\left(\frac{c g_{b} w_{b}}{2 N_{b}}\right)+\frac{g_{b f} N_{f} w_{b}^{4}}{\sqrt{\pi}\left(w_{b}^{2}+w_{f}^{2}\right)^{3 / 2}} \\
& =0 \\
1 & +\frac{2 N_{f}^{2}}{\sqrt{3} \pi} F\left(\frac{c g_{f} w_{f}}{2 N_{f}}\right)-\frac{N_{f} g_{f} w_{f}}{2 \sqrt{2 \pi}} F^{\prime}\left(\frac{c g_{f} w_{f}}{2 N_{f}}\right)+\frac{g_{b f} N_{b} w_{f}^{4}}{\sqrt{\pi}\left(w_{b}^{2}+w_{f}^{2}\right)^{3 / 2}} \\
& =0 .
\end{aligned}
$$

The remaining variational equations are $\partial L / \partial A_{j}=0$, which yield $\mu$ as a function of $w_{j}$ 's, and $g$ 's:

$$
\begin{aligned}
\mu_{b}= & \frac{1}{2 w_{b}^{2}}-\frac{N_{b} g_{b}}{2 w_{b} \sqrt{2 \pi}} G^{\prime}\left(\frac{c g_{b} w_{b}}{2 N_{b}}\right)+\frac{\sqrt{3} N_{b}^{2}}{\pi w_{b}^{2}} G\left(\frac{c g_{b} w_{b}}{2 N_{b}}\right) \\
& +\frac{g_{b f} N_{f}}{\sqrt{\pi\left(w_{f}^{2}+w_{b}^{2}\right)}}, \\
\mu_{f}= & \frac{1}{2 w_{f}^{2}}+\frac{\sqrt{3} N_{f}^{2}}{\pi w_{f}^{2}} F\left(\frac{c g_{f} w_{f}}{2 N_{f}}\right)-\frac{N_{f} g_{f}}{2 w_{f} \sqrt{2 \pi}} F^{\prime}\left(\frac{c g_{f} w_{f}}{2 N_{f}}\right) \\
& +\frac{g_{b f} N_{b}}{\sqrt{\pi\left(w_{f}^{2}+w_{b}^{2}\right)}} .
\end{aligned}
$$

Equations (44)-(47) are the variational results which we shall use in our study of bright Bose-Fermi solitons.

\section{B. Numerical results}

For stationary solutions we solve time-independent equations (11) and (12) by using an imaginary-time propagation method based on the finite-difference Crank-Nicholson dis- cretization scheme of time-dependent equations (9) and (10). The nonequilibrium dynamics from an initial stationary state is studied by solving the time-dependent equations (9) and (10) with real-time propagation by using as initial input the solution obtained by the imaginary-time propagation method. The reason for this mixed treatment is that the imaginarytime propagation method deals with real variables only and provides a very accurate solution of the stationary problem at low computational cost [64]. In the finite-difference discretization we use a space step of 0.025 and time step of 0.0005 .

First we report results for stationary profiles of the localized Bose and Fermi solitons formed in the presence of attractive Bose-Fermi and Fermi-Fermi interactions and repulsive Bose-Bose interactions. In the presence of weak attractive Fermi-Fermi interactions, the fermions form a BCS state which satisfies a nonlinear Schrödinger equation with repulsive (self-defocusing) quintic nonlinearity. As the strength of the attractive Fermi-Fermi interaction increases the fermions pass from the BCS regime to the unitarity regime which is described by another nonlinear Schrödinger equation with repulsive (self-defocusing) nonlinearity. Hence fermions cannot form a bright soliton by itself. However, they can form a bright soliton in the presence of an attractive Bose-Fermi interaction.

If the fermionic repulsive nonlinearity is not very large, bosons and fermions form mostly overlapping (mixed) solitons both in the BCS and unitarity regimes. Note that in the BCS and unitarity regimes the fermionic system becomes repulsive in the presence of a Fermi-Fermi attraction. However, as the fermionic repulsive nonlinearity turns large, the fermionic profile comes out of the bosonic profile and partially demixed solitons are created. We studied the numerically calculated soliton profiles for a wide range of BoseBose, Bose-Fermi, and Fermi-Fermi interactions and boson and fermion numbers solving Eqs. (11) and (12) by the technique of imaginary-time propagation and compared them with the Gaussian variational results obtained from Eqs. (44) and (45). Except in the case of strong demixing, when the fermion profile strongly deviates from Gaussian shape, the agreement between variational and numerical profiles is quite good.

In Fig. 3 we present typical soliton profiles illustrating the change in the results during BCS-unitarity crossover as well as the demixed profiles. In Figs. 3(a)-3(c) we show the soliton profiles for weak (BCS phase), moderate, and strong (unitarity regime) Fermi-Fermi attraction corresponding to strengths $g_{f}=-0.1,-1$, and -10 , respectively, for $g_{b}=0.01$, $g_{b f}=-0.4, N_{f}=20$, and $N_{b}=300$ and compare these with the variational results. Figure 3(d) illustrates a demixed state obtained by increasing the fermion number from the configuration of Fig. 3(c) from $N_{f}=20$ to 100. In this case the (numerically obtained) fermion profile stretches far beyond the bosonic profile and is poorly represented by a Gaussian shape, which is the cause of the deviation of the variational result from the numerical result.

After illustrating the soliton profiles in different states it is now pertinent to verify if these solitons are dynamically stable under perturbation. To this end we consider the typical stationary soliton of Fig. 3(a) (obtained by the imaginarytime propagation method) and subject it to the perturbation 

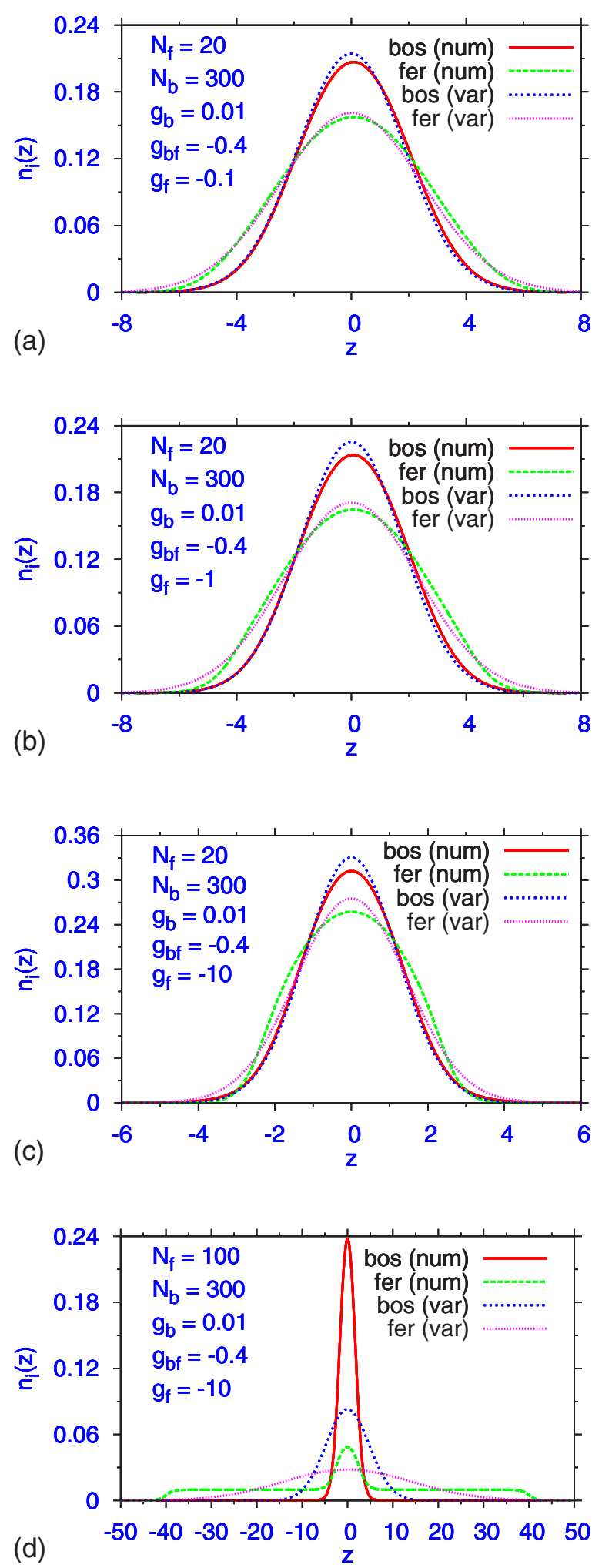

FIG. 3. (Color online) Probability densities from numerical solution of Eqs. (9) and (10) [here normalized to unity: $\int_{-\infty}^{\infty} n_{i}(z) d z$ $=1$ ] compared with variational results given by Eqs. (44) and (45) for $N_{b}=300, g_{b}=0.01, g_{b f}=-0.4$, and (a) $N_{f}=20, g_{f}=-0.1$, (b) $N_{f}$ $=15, g_{f}=-1$, (c) $N_{f}=20, g_{f}=-10$, and (d) $N_{f}=100, g_{f}=-10$. Of these, (a) represents fermions in the BCS regime, (b) represents fermions in the BCS-to-unitarity crossover, (c) represents fermions in the unitarity regime, and (d) represents bosons and fermions in a partially demixed configuration.
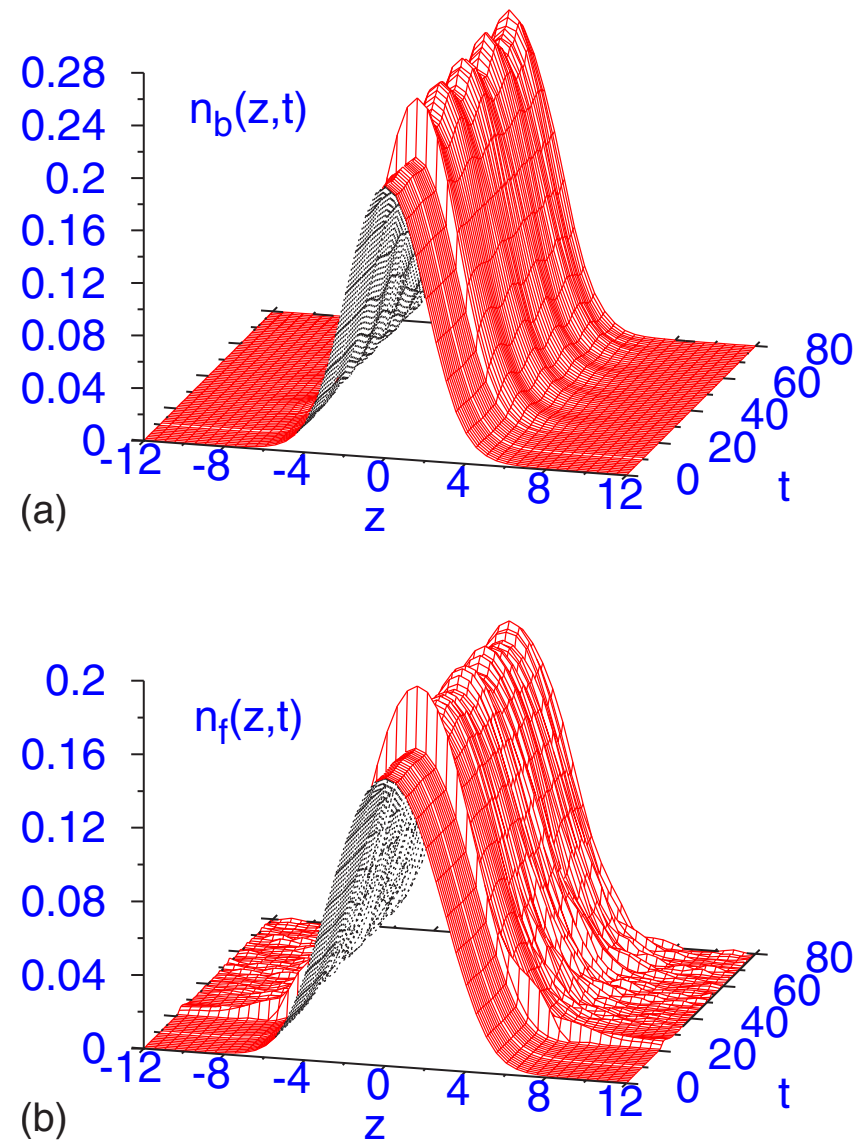

FIG. 4. (Color online) Dynamics of the probability density profiles of (a) Bose and (b) Fermi solitons of Fig. 3(a) when at $t=20$ they are subject to a quite strong perturbation by setting $\phi_{j}(z, t)$ $=1.1 \phi_{j}(z, t)$. The solitons undergo stable propagation as long as we could continue the numerical simulation. The initial soliton profile is calculated with an imaginary-time propagation algorithm and the dynamics studied with a real-time propagation algorithm. The soliton profiles are normalized to unity: $\int_{-\infty}^{\infty} n_{j}(z, t) d z=1$.

$\phi_{j}(z, t)=1.1 \phi_{j}(z, t)$ and observe the resultant dynamics (obtained by the real-time propagation method), which is illustrated in Fig. 4. The solitons under this perturbation execute breathing oscillations and propagate for as long as the numerical simulation was continued without being destroyed. This demonstrates the stability of the solitons under perturbation.

Finally, in Fig. 5 we show the chemical potential for $\mu_{b}$ bosons and $\mu_{f}$ for fermions as a function of Fermi-Fermi interaction strength $g_{f}$ for $N_{f}=20, N_{b}=300, g_{b}=0.01$, and $g_{b f}=-0.4$ and -0.8 obtained from numerical solution and Gaussian variational analysis. The agreement between the two results for $\mu_{b}$ is good whereas for $\mu_{f}$ is only fair. The increase of the Fermi-Fermi attraction strength $\left|g_{f}\right|$ for a fixed $g_{b f}$ corresponds to a reduction in both chemical potentials, signaling strongly bound solitons. The same happens for the increase of Bose-Fermi attraction strength $\left|g_{b f}\right|$ from 0.4 to 0.8. In Fig. 5 the small- $\left|g_{f}\right|$ limit corresponds to the BCS phase of bosons whereas the large- $\left|g_{f}\right|$ limit corresponds to the unitarity regime of fermions. The intermediate values of $\left|g_{f}\right|$ denote the crossover from BCS to the unitarity regime. 

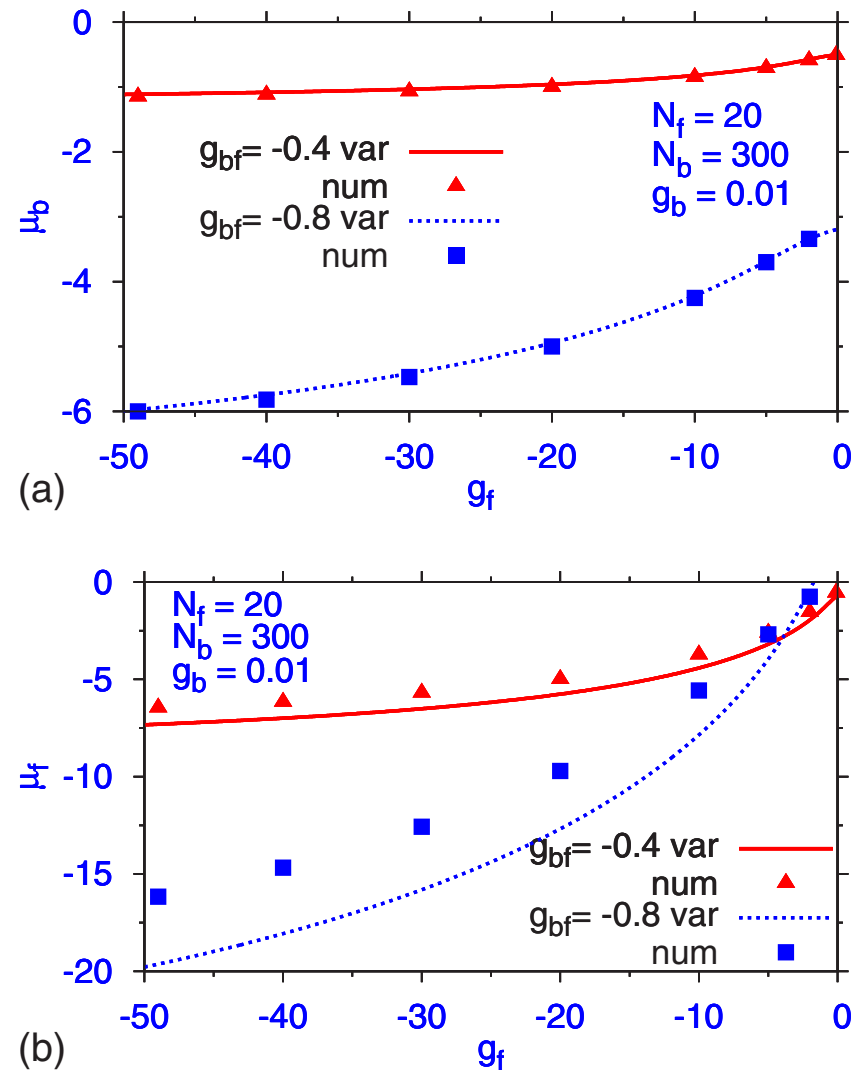

FIG. 5. (Color online) Chemical potentials for (a) bosons and (b) fermions vs Fermi-Fermi interaction strength $g_{f}$ for $N_{f}=20$, $N_{b}=300, g_{b}=0.01$ for $g_{b f}=-0.4$ and -0.8 calculated numerically (labeled "num" shown by symbols) compared with variational results (labeled "var" shown by solid lines).

\section{SUMMARY AND CONCLUSION}

In this paper we have studied a one-dimensional superfluid Bose-Fermi mixture using a set of coupled mean-field equations derived as the Euler-Lagrange equation employing the Lieb-Liniger energy density of bosons and the GaudinYang energy density of fermions and an interaction term proportional to the product of probability density of bosons and fermions. This set of coupled equations has a complex nonlinearity structure for fermions and bosons and shows the proper transition from a cubic Bose nonlinearity in the weakcoupling GP BCS limit to a quintic nonlinearity in the strong-coupling TG (Tonks-Girardeau) limit. In addition, for fermions with attractive interactions considered in this paper, it shows the proper transition from the weak-coupling BCS regime to unitarity limit: both limits are described by a quintic nonlinearity with different coefficients. In this model, in the extreme weak-coupling BCS limit the superfluid fermionic energy density is identical to that of a noninteracting degenerate Fermi gas in the normal state [52].

We consider two distinct situations for the superfluid Bose-Fermi mixture: (i) in a ring with periodic boundary condition realizable from a toroidal trap in the limit of strong transverse confinement and (ii) in an infinite cylinder with open boundary condition realizable from an axially symmetric trap in the limit of strong transverse and zero axial confinement.

For the mixture in a ring, from energetic considerations, we obtain the condition of stability of a uniform mixture with a constant probability density. The uniform mixture is energetically stable for interspecies attraction strength $\left|g_{b f}\right|$ below a critical value, above which stable lowest-energy states are bright Bose-Fermi solitons. For the repulsive interspecies interaction the stable lowest-energy states are demixed states of Bose-Fermi mixture, where the region of a maximum of boson probability density corresponds to a minimum of fermion probability density. It is also demonstrated algebraically that for attractive Bose-Fermi interaction the bright solitons can be created from the uniform mixture above the critical Bose-Fermi attraction by modulational instability of the uniform mixture under a weak perturbation.

In the one-dimensional infinite cylinder we solved the coupled set of equations for the superfluid Bose-Fermi mixture numerically and using a Gaussian variational approximation. We calculated numerically the probability density profiles of the bright solitons as well as their chemical potentials and compared them with the respective Gaussian variational approximations. The agreement between the two is good to fair. In this case a partial demixing of the BoseFermi solitons is possible, when the Fermi soliton extends over a very large region in space while the Bose soliton remains fairly localized. We also established numerically the dynamical stability of the Bose-Fermi solitons by inflicting a perturbation on the solitons by multiplying the wavefunction profiles by 1.1 . The system is then found to propagate over a very long period of time executing breathing oscillation without being destroyed, which demonstrated the stability of the solitons. Finally, we comment that in view of the experimental realization of the superfluid Bose-Fermi mixture [45] and observation of solitons in a pure BEC $[36,37]$, the achievement of a bright Bose-Fermi soliton seems possible through a controlled manipulation of strengths of atomic interactions by varying an external background magnetic field near a Feshbach resonance [47] and by adopting the set of parameters we have used in this paper.

\section{ACKNOWLEDGMENTS}

S.K.A. thanks FAPESP and CNPq for partial financial support. L.S. acknowledges partial financial support by the Italian GNFM-INdAM through the project "Giovani Ricercatori" and by Fondazione CARIPARO. 
[1] M. Takahashi, Thermodynamics of One-Dimensional Solvable Models (Cambridge University Press, Cambridge, England, 1999).

[2] A. J. Leggett, Quantum Liquids: Bose Condensation and Cooper Pairing in Condensed-Matter Systems (Oxford University Press, Oxford, 2006).

[3] T. Kinoshita, T. Wenger, and D. S. Weiss, Science 305, 1125 (2004).

[4] B. Paredes et al., Nature (London) 429, 277 (2004).

[5] M. Girardeau, J. Math. Phys. 1, 516 (1960).

[6] E. H. Lieb and W. Liniger, Phys. Rev. 130, 1605 (1963).

[7] D. S. Petrov, G. V. Shlyapnikov, and J. T. M. Walraven, Phys. Rev. Lett. 85, 3745 (2000); V. Dunjko, V. Lorent, and M. Olshanii, ibid. 86, 5413 (2001).

[8] P. Öhberg and L. Santos, Phys. Rev. Lett. 89, 240402 (2002).

[9] E. H. Lieb, R. Seiringer, and J. Yngvason, Phys. Rev. Lett. 91, 150401 (2003); Commun. Math. Phys. 244, 347 (2004).

[10] L. Salasnich, A. Parola, and L. Reatto, Phys. Rev. A 72, 025602 (2005).

[11] L. Salasnich, Laser Phys. 12, 198 (2002); L. Salasnich, A. Parola, and L. Reatto, Phys. Rev. A 65, 043614 (2002).

[12] L. Salasnich, A. Parola, and L. Reatto, Phys. Rev. A 69, 045601 (2004); L. Salasnich, J. Phys. B 39, 1743 (2006).

[13] L. Salasnich, A. Parola, and L. Reatto, Phys. Rev. A 70, 013606 (2004).

[14] M. Greiner, C. A. Regal, and D. S. Jin, Nature (London) 426, 537 (2003)

[15] C. A. Regal, M. Greiner, and D. S. Jin, Phys. Rev. Lett. 92, 040403 (2004).

[16] J. Kinast, S. L. Hemmer, M. E. Gehm, A. Turlapov, and J. E. Thomas, Phys. Rev. Lett. 92, 150402 (2004).

[17] M. W. Zwierlein, C. A. Stan, C. H. Schunck, S. M. F. Raupach, A. J. Kerman, and W. Ketterle, Phys. Rev. Lett. 92, 120403 (2004); M. W. Zwierlein, C. H. Schunck, C. A. Stan, S. M. F. Raupach, and W. Ketterle, Phys. Rev. Lett. 94, 180401 (2005).

[18] C. Chin et al., Science 305, 1128 (2004); M. Bartenstein, A. Altmeyer, S. Riedl, S. Jochim, C. Chin, J. H. Denschlag, and R. Grimm, ibid. 92, 203201 (2004).

[19] L. D. Landau and E. M. Lifshitz, Quantum Mechanics-Non Relativistic Theory, Course of Theoretical Physics, Vol. 3 (Pergamon Press, New York, 1989).

[20] M. Gaudin, Phys. Lett. 24A, 55 (1967); C. N. Yang, Phys. Rev. Lett. 19, 1312 (1967).

[21] S. Tomonaga, Prog. Theor. Phys. 5, 544 (1950); J. M. Luttinger, J. Math. Phys. 4, 1154 (1963).

[22] A. Luther and V. J. Emery, Phys. Rev. Lett. 33, 589 (1974).

[23] J. N. Fuchs, A. Recati, and W. Zwerger, Phys. Rev. Lett. 93, 090408 (2004).

[24] I. V. Tokatly, Phys. Rev. Lett. 93, 090405 (2004).

[25] A. G. Truscott et al., Science 291, 2570 (2001); G. Modugno, G. Roati, F. Riboli, F. Ferlaino, R. J. Brecha, and M. Inguscio, ibid. 297, 2240 (2002).

[26] F. Schreck, L. Khaykovich, K. L. Corwin, G. Ferrari, T. Bourdel, J. Cubizolles, and C. Salomon, Phys. Rev. Lett. 87, 080403 (2001).

[27] Z. Hadzibabic, C. A. Stan, K. Dieckmann, S. Gupta, M. W. Zwierlein, A. Gorlitz, and W. Ketterle, Phys. Rev. Lett. 88, 160401 (2002).

[28] B. DeMarco and D. S. Jin, Science 285, 1703 (1999); G. Roati, F. Riboli, G. Modugno, and M. Inguscio, Phys. Rev.
Lett. 89, 150403 (2002).

[29] K. Mölmer, Phys. Rev. Lett. 80, 1804 (1998); R. Roth, Phys. Rev. A 66, 013614 (2002); P. Capuzzi, A. Minguzzi, and M. P. Tosi, ibid. 67, 053605 (2003); M. Modugno, F. Ferlaino, F. Riboli, G. Roati, G. Modugno, and M. Inguscio, ibid. 68, 043626 (2003).

[30] N. Nygaard and K. Mølmer, Phys. Rev. A 59, 2974 (1999).

[31] M. J. Bijlsma, B. A. Heringa, and H. T. C. Stoof, Phys. Rev. A 61, 053601 (2000).

[32] H. Heiselberg, C. J. Pethick, H. Smith, and L. Viverit, Phys. Rev. Lett. 85, 2418 (2000).

[33] L. Viverit, C. J. Pethick, and H. Smith, Phys. Rev. A 61, 053605 (2000); L. Viverit, ibid. 66, 023605 (2002).

[34] K. K. Das, Phys. Rev. Lett. 90, 170403 (2003).

[35] L. Salasnich and F. Toigo, Phys. Rev. A 75, 013623 (2007).

[36] K. E. Strecker, G. B. Partridge, A. G. Truscott, and R. G. Hulet, Nature (London) 417, 150 (2002); L. Khaykovich, F. Schreck, G. Ferrari, T. Bourdel, J. Cubizolles, L. D. Carr, Y. Castin, and C. Salomon, Science 256, 1290 (2002); V. M. Pérez-García, H. Michinel, and H. Herrero, Phys. Rev. A 57, 3837 (1998).

[37] S. L. Cornish, S. T. Thompson, and C. E. Wieman, Phys. Rev. Lett. 96, 170401 (2006).

[38] K. E. Strecker, G. B. Partridge, A. G. Truscott, and R. G. Hulet, New J. Phys. 5, 73 (2003); V. A. Brazhnyi and V. V. Konotop, Mod. Phys. Lett. B 18, 627 (2004); F. Kh. Abdullaev, A. Gammal, A. M. Kamchatnov, and L. Tomio, Int. J. Mod. Phys. B 19, 3415 (2005); V. I. Yukalov, Laser Phys. Lett. 1, 435 (2004); A. Minguzzi, S. Succi, F. Toschi, M. P. Tosi, and P. Vignolo, Phys. Rep. 395, 223 (2004).

[39] T. Karpiuk, K. Brewczyk, S. Ospelkaus-Schwarzer, K. Bongs, M. Gajda, and K. Rzazewski, Phys. Rev. Lett. 93, 100401 (2004); T. Karpiuk, M. Brewczyk, and K. Rzazewski, Phys. Rev. A 73, 053602 (2006).

[40] S. K. Adhikari, Phys. Rev. A 72, 053608 (2005).

[41] S. K. Adhikari, J. Phys. A 40, 2673 (2007); Eur. Phys. J. D 40, 157 (2006); Laser Phys. Lett. 3, 605 (2006); J. Phys. B 38, 3607 (2005); I. Kourakis et al., Eur. Phys. J. B 46, 381 (2005).

[42] S. K. Adhikari, Phys. Lett. A 346, 179 (2005); V. M. PérezGarcía and J. B. Beitia, Phys. Rev. A 72, 033620 (2005).

[43] S. K. Adhikari and L. Salasnich, Phys. Rev. A 75, 053603 (2007).

[44] S. K. Adhikari, Phys. Rev. A 73, 043619 (2006); S. K. Adhikari and B. A. Malomed, ibid. 74, 053620 (2006).

[45] M. W. Zwierlein, J. R. Abo-Shaeer, A. Schirotzek, C. H. Schunck, and W. Ketterle, Nature (London) 435, 1074 (2005).

[46] D. M. Eagles, Phys. Rev. 186, 456 (1969); A. J. Leggett, J. Phys. (Paris), Colloq. 41, C7-19 (1980); M. Casas, J. M. Getino, M. de Llano, A. Puente, R. M. Quick, H. Rubio, and D. M. van dev Walt, Phys. Rev. B 50, 15945 (1994); S. K. Adhikari, M. Casas, A. Puente, A. Rigo, M. Fortes, M. A. Solis, M. de Llano, A. A. Valladaves, and O. Rajo, ibid. 62, 8671 (2000); Physica C 453, 37 (2007).

[47] S. Inouye, M. R. Andrews, J. Stenger, H. J. Miesner, D. M. Stamper-Kurn, and W. Ketterle, Nature (London) 392, 151 (1998).

[48] G. M. Kavoulakis, Phys. Rev. A 67, 011601(R) (2003); R. Kanamoto, H. Saito, and M. Ueda, ibid. 67, 013608 (2003).

[49] R. Kanamoto, H. Saito, and M. Ueda, Phys. Rev. A 68, 043619 (2003); G. M. Kavoulakis, ibid. 69, 023613 (2004). 
[50] L. Salasnich, A. Parola, and L. Reatto, Phys. Rev. A 59, 2990 (1999); A. Parola, L. Salasnich, R. Rota, and L. Reatto, ibid. 72, 063612 (2005); L. Salasnich, A. Parola, and L. Reatto, ibid. 74, 031603(R) (2006).

[51] S. Gupta, K. W. Murch, K. L. Moore, T. P. Purdy, and D. M. Stamper-Kurn, Phys. Rev. Lett. 95, 143201 (2005).

[52] L. Salasnich, S. K. Adhikari, and F. Toigo, Phys. Rev. A 75, 023616 (2007).

[53] M. Olshanii, Phys. Rev. Lett. 81, 938 (1998).

[54] The Lieb-Liniger function $G(x)$ is such that for $x \ll 1$ one finds $G(x) \simeq x+B x^{3 / 2}+\eta x^{2}$, where $B=4 /(3 \pi)$ and $\eta=0.0648$ (from numerics). Instead for $x \gg 1$ one has $G(x) \simeq\left(\pi^{2} / 2\right) x^{2} /(x+2)^{2}$. $G(x)$ can be very well described by the following Padè approximant: $\quad G(x)=\left(x+A x^{3}\right) /\left[1+B x^{1 / 2}+C x(x+2)^{2}\right]$, where $A$ $=\pi^{2}\left(B^{2}-\eta\right) / 12$ and $C=\left(B^{2}-\eta\right) / 4$.

[55] The Gaudin-Yang function $F(x)$ is conveniently parametrized by the following Padè approximant: $F(x)=\left(\pi^{2} / 48\right)\left(x^{2}-x\right.$ $+3 / 4) /\left(x^{2}+q_{1} x+q_{2}\right)$, where $q_{1}=-\left[9 /\left(8 \pi^{2}\right)+1 / 4\right]=-0.3633$ and $q_{2}=3 / 16$.

[56] L. Salasnich, J. Math. Phys. 41, 8016 (2000); L. Salasnich, B. Pozzi, A. Parola, and L. Reatto, J. Phys. B 33, 3943 (2000).

[57] E. Cerboneschi, R. Mannella, E. Arimondo, and L. Salasnich, Phys. Lett. A 249, 495 (1998); L. Salasnich, A. Parola, and L. Reatto, Phys. Rev. A 64, 023601 (2001).
[58] A. S. Alexandrov and V. V. Kabanov, J. Phys.: Condens. Matter 14, L327 (2002).

[59] V. V. Konotop and M. Salerno, Phys. Rev. A 65, 021602(R) (2002); L. Salasnich, A. Parola, and L. Reatto, Phys. Rev. Lett. 91, 080405 (2003).

[60] L. Salasnich, N. Manini, F. Bonelli, M. Korbman, and A. Parola, Phys. Rev. A 75, 043616 (2007).

[61] B. A. Malomed, in Progress in Optics edited by E. Wolf (North-Holland, Amsterdam, 2002), Vol. 43, p. 71; V. M. Pérez-García, H. Michinel, J. I. Cirac, M. Lewenstein, and P. Zoller, Phys. Rev. A 56, 1424 (1997).

[62] L. Salasnich, Mod. Phys. Lett. B 11, 1249 (1997); A. Parola, L. Salasnich, and L. Reatto, Phys. Rev. A 57, R3180 (1998); L. Salasnich, Mod. Phys. Lett. B 12, 649 (1998).

[63] L. Reatto, A. Parola, and L. Salasnich, J. Low Temp. Phys. 113, 195 (1998); L. Salasnich, Phys. Rev. A 61, 015601 (2000); Int. J. Mod. Phys. B 14, 1 (2000); S. K. Adhikari, Phys. Rev. E 71, 016611 (2005); J. Phys. B 38, 579 (2005).

[64] S. K. Adhikari and P. Muruganandam, J. Phys. B 35, 2831 (2002); S. K. Adhikari, Phys. Rev. A 69, 063613 (2004); P. Muruganandam and S. K. Adhikari, J. Phys. B 36, 2501 (2003); S. K. Adhikari, Phys. Lett. A 265, 91 (2000); Phys. Rev. E 62, 2937 (2000);Phys. Rev. C 19, 1729 (1979). 